\title{
Akuter Husten hat oft eine chronische Ursache
}

- Jeder fünfte Patient, der sich wegen akuten Hustens beim Hausarzt vorstellt, hat Auffälligkeiten in der Spirometrie, die auf ein bisher nicht entdecktes Asthma oder eine nicht erkannte COPD hindeuten. Das zeigt eine Studie, an der Primärärzte aus zwölf europäischen Ländern, darunter auch Deutschland, beteiligt waren. Die Ärzte hatten 1947 konsekutive erwachsene Patienten mit einem kürzer als 28 Tage dauernden Husten und ohne COPD- und Asthmadiagnose für die Studie gewonnen. Die Patienten waren im Mittel 50 Jahre alt und hatten vor dem Hausarztbesuch bereits neun Tage gehustet. Vier Wochen nach der Erstvorstellung, wenn der Husten wieder verschwunden war, wurde bei allen Patienten die Lungenfunktion geprüft.

Das Ergebnis: Bei 240 Studienteilnehmern (12\%) fanden sich Hinweise auf ein unerkanntes Asthma. Sie hatten einen positiven Bronchospasmolyse-Test mit einer $\mathrm{FEV}_{1}$-Zunahme um mindestens $12 \%$ und um mehr als $200 \mathrm{ml}$. Außerdem berichteten sie, im vergangenen Jahr mindestens

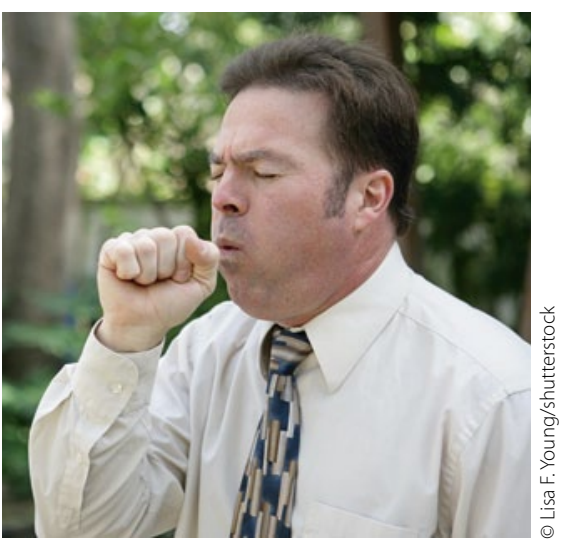

Nur ein Erkältungshusten - oder steckt eine COPD dahinter?

eine weitere Episode von Giemen, Husten oder Engegefühl im Thorax durchgemacht zu haben. Patienten unter 50 Jahren waren häufiger betroffen als ältere (15\% vs. $11 \%$ ). Anzeichen einer persistierenden Atemwegsobstruktion wurden bei bis zu 10\% der Patienten entdeckt. 55\% der Patienten mit einer $\mathrm{FEV}_{1}$ : $\mathrm{FVC}<0,7$ hatten eine $\mathrm{FEV}_{1}$ unter $80 \%$ des erwarteten Wertes, bei $8 \%$ der Patienten lag sie sogar schon unter $50 \%$.

Erwartungsgemäß wurde eine Obstruktion nach GOLD häufiger bei Patienten ab 50 als bei jüngeren Patienten nachgewiesen ( $14 \%$ vs. $6 \%$ ). Raucher waren überraschenderweise nicht überdurchschnittlich häufig vertreten. Das mag mit einer teilweise unvollständigen Dokumentation des Raucherstatus zusammenhängen, aber auch damit, dass rauchende COPD-Patienten entweder früher erkannt oder seltener in der Praxis gesehen werden.

Damit lieferte die Spirometrie bei jedem fünfte Patienten mit akutem Husten Hinweise auf eine bisher nicht bekannte chronische Atemwegserkrankung. Insbesondere bei der COPD geht man heute von einer massiven Unterdiagnose aus: Bis zu 50\% der Patienten sollen nicht erkannt sein; geschätzt wird, dass in Deutschland mehr als $10 \%$ der über 40 -Jährigen betroffen sind.

BS =

Van Vugt S et al. Ann Fam Med 2012; 10: 523-529; doi:10.1370/afm.1416

\section{Hepatitis-Index für Europa veröffentlicht}

Ein europäischer Hepatitis-Index erlaubt erstmals einen Überblick über die Präventions- und Versorgungssituation in Bezug auf Hepatitis B und C in 30 europäischen Ländern.

Beim Euro Hepatitis Index handelt es sich um eine Beobachtungsstudie im Auftrag der europäischen Patientenorganisation ELPA (European Liver Patients Association) in 27 EU-Mitgliedsländern sowie Norwegen, Kroatien und der Schweiz. Regierungsvertreter, Hepatologen und nationale Patientenorganisationen sollten einen von Experten erstellten Katalog von 31 Fragen beantworten. Diese beschäftigen sich mit der Prävention, dem Zugang zu Therapien, dem Vorhandensein nationaler HepatitisStrategien sowie mit epidemiologischen Daten und Behandlungsresultaten.

\section{Große regionale Unterschiede}

Daraus ergibt sich ein sehr heterogenes Bild. So schneiden zwar Länder wie Frankreich, Slowenien und Deutschland, gemessen an den vergebenen Punktwerten, vergleichsweise gut ab. Allerdings liegt selbst in Ländern mit nationalen Hepatitis-Plänen wie Frankreich und Schottland die Hepatitis-Detektionsrate unter $40 \%$. Frankreich wird besonders für seine Kampagnen gelobt, um öffentlich Aufmerksamkeit für Virushepatitiden herzustellen sowie für das dichte Netzwerk von Referenzzentren.
Nationaler Hepatitis-Plan angekündigt Deutschland erhält gute Noten, unter anderem für den guten Zugang zu modernen Therapieoptionen. „Wir haben ein Luxusproblem in Deutschland", sagt Achim Kautz, Geschäftführer der Deutschen Leberhilfe e.V. Einerseits bestehe eine sehr gute fachärztliche Versorgung, vergleichsweise kurze Wartezeiten sowie Zugang zu den neuesten Therapeutika. Andererseits fehle es an öffentlicher Aufmerksamkeit für die Hepatitis-Problematik sowohl in der Bevölkerung als auch in Fachkreisen, und die Budgetierung verhindere die optimale Versorgung. Kautz kündigte einen nationalen Hepatitis-Plan für Deutschland an, initiiert von Patienten- und Fachorganisationen. $\mathrm{Er}$ soll noch dieses Jahr vorgestellt werden. Infos im Internet: www.elpa-info.org 